\title{
Pathogenesis-related protein 1 (PR-1) genes in soybean: genome-wide identification, structural analysis and expression profiling under multiple biotic and abiotic stresses
}

\author{
Fabricio Almeida-Silva ${ }^{1}$ and Thiago M. Venancio ${ }^{1 *}$
}

${ }^{1}$ Laboratório de Química e Função de Proteínas e Peptídeos, Centro de Biociências e Biotecnologia, Universidade Estadual do Norte Fluminense Darcy Ribeiro, Campos dos Goytacazes, RJ, Brazil.

*FA-S: Laboratório de Química e Função de Proteínas e Peptídeos, Centro de Biociências e Biotecnologia, Universidade Estadual do Norte Fluminense Darcy Ribeiro. Av. Alberto Lamego 2000, P5, sala 217, Campos dos Goytacazes, RJ, Brazil. Email: fabricio almeidasilva@hotmail.com

*TMV: Laboratório de Química e Função de Proteínas e Peptídeos, Centro de Biociências e Biotecnologia, Universidade Estadual do Norte Fluminense Darcy Ribeiro. Av. Alberto Lamego 2000, P5, sala 217, Campos dos Goytacazes, RJ, Brazil. Email: thiago.venancio@gmail.com 


\section{ABSTRACT}

2 Plant pathogenesis-related (PR) proteins are a large group of proteins, classified in 17

3 families, that are induced by pathological conditions. Here, we characterized the soybean PR-

41 (GmPR-1) gene repertoire at the sequence, structural and expression levels. We found 24

5 GmPR-1 genes, clustered in two phylogenetic groups. GmPR-1 genes are under strong

6 purifying selection, particularly those that emerged by tandem duplications. GmPR-1

7 promoter regions are abundant in cis-regulatory elements associated with major stress-

8 related transcription factor families, namely WRKY, ERF, HD-Zip, C2H2, NAC, and GATA. We

9 observed that 23 GmPR-1 genes are induced by stress conditions or exclusively expressed

10 upon stress. We explored 1972 transcriptome samples, including 26 stress conditions,

11 revealing that most GmPR-1 genes are differentially expressed in a plethora of biotic and

12 abiotic stresses. Our findings highlight stress-responsive GmPR-1 genes with potential

13 biotechnological applications, such as the development of transgenic lines with increased

14 resistance to biotic and abiotic stresses.

15

16 Keywords: plant immunity, phytopathology, gene family evolution, functional genomics. 


\section{Introduction}

Plants have evolved a complex genetic machinery to cope with pathogens and pests. Plant pathogenesis-related (PR) proteins are core components of plant defense and are classified in 17 families based on their sequence similarities, enzymatic activities and structural properties [1]. PR-1, the first identified family of PR proteins, was discovered in tobacco leaves infected with tobacco mosaic virus [2]. Alexander et al. [2] reported that accumulated PR-1 proteins comprised $2 \%$ of the total proteins in infected tobacco leaves, suggesting a 24 prominent role in plant defense.

PR-1 proteins are ubiquitous and have been identified in many plant species [3-6]. They can be acidic or alkaline and are mostly secreted to the apoplast, although some can be stored in vacuoles [7]. All PR-1 proteins share a conserved CAP (cysteine-rich secretory protein, antigen 5 , pathogenesis-related 1 ) domain that typically folds into $4 \alpha$-helices and 4$\beta$ sheets stabilized by disulfide bonds [8]. These unique features are responsible for their biological functions, which are mainly associated with defense to biotic and abiotic stresses $[4,9]$. However, some works have also linked PR-1 proteins with endogenous functions, such as leaf senescence and floral development [10].

Soybean (Glycine max (L.) Merr.) is the most important legume crop worldwide, being used in human and animal nutrition, and industrial applications. Soybean diseases are responsible for an economic loss of 4.6 billion dollars only in the US [11]. Likewise, soybean yield is significantly affected by multiple environmental factors [12]. Transgenic plants overexpressing PR-1 genes have demonstrated increased resistance to both biotic and abiotic stresses $[5,13,14]$. However, the soybean PR-1 gene repertoire has never been systematically characterized. Hence, a comprehensive analysis of the soybean PR-1 genes and their transcriptional profiles under multiple stresses would certainly be useful to pinpoint potential

41 targets for biotechnological applications, such as developing transgenic lines with increased 42 resistance.

Here, we have characterized the soybean PR-1 (GmPR-1) gene repertoire at the 44 sequence, structural and transcriptional levels, under diverse tissues and stress conditions. 45 We found 24 GmPR-1 genes clustered in two phylogenetic groups. All GmPR-1 genes are 46 under strong purifying selection, particularly tandem-derived paralogs, and they tend to have 47 tissue-specific expression. Besides, we observed that major stress-related transcription factor 48 (TF) families (e.g., WRKY, $\mathrm{C} 2 \mathrm{H} 2$, and ERF) are putative regulators of GmPR-1 gene 49 expression. Finally, we demonstrated that most GmPR-1 genes are transcriptionally 50 modulated by a plethora of biotic and abiotic stresses, from which one is exclusively expressed 
51 upon stress. We reported the transcriptional landscape of GmPR-1 genes under 26 different

52 biotic and abiotic stress conditions, making this study the most comprehensive investigation

53 of GmPR-1 transcriptional response to stresses. Our findings can help select potential GmPR-

541 genes to be used in the development of transgenic lines with increased stress resistance.

562 Materials and Methods

572.1 Identification of GmPR-1 genes

58 Soybean predicted proteins were downloaded from the PLAZA database [15]. GmPR-1 59 proteins were identified with BLASTp searches [16] against the soybean predicted proteins 60 (E-value threshold: 1e-10) using curated Arabidopsis thaliana PR-1 proteins as queries 61 (UniProt IDs: P33154 and Q9ZNS4). The proteins found in the BLASTp search were then 62 scanned for the presence of the CAP domain (PF00188). Further, we confirmed that all GmPR631 genes were included in the same subfamily in PLAZA (ORTHO04D000086) [15]. Functional 64 gene annotations (Gene Ontology and PFAM) were retrieved from SoyBase [17] and PLAZA 65 [15].

\subsection{Homology modelling and structural analysis}

Tertiary protein structures and the percentage of secondary structures were predicted with the Phyre2 server [18]. Physicochemical properties were identified with the R package Peptides [19]. Signal peptides were predicted with SignalP 5.0 [20]. The .gff file containing exon-intron boundaries of GmPR-1 genes was downloaded from PLAZA [15] and visualized with gggenes [21]. Protein length calculation and sequence manipulations were performed with Biostrings [22].

\subsection{Phylogenetic reconstruction, gene duplication and selection analyses}

76 Protein sequences were aligned with MAFFT [23] and visualized with msa [24]. Maximum

77 likelihood phylogenetic trees were inferred using IQTREE2 [25] with 1000 bootstraps. A

78 BLASTP search against the UniProt database [26] was performed to identify an outgroup for 79 the tree of GmPR-1 genes, and a Cajanus cajan PR-1 (UniProt ID: A0A151S132) was selected 80 as the best hit after excluding G. max hits. PR-1 proteins from $A$. thaliana and Vigna radiata 81 were retrieved from PLAZA by searching for genes in the same subfamily of GmPR-1 genes 
82 (ORTHO04D000086). The R package ggtree [27] was used for tree visualization. Paralogous 83 gene pairs and their ratios of the number of nonsynonymous substitutions per

84 nonsynonymous substitution site to the number of synonymous substitutions per synonymous

85 substitution site $(\mathrm{Ka} / \mathrm{Ks})$ were retrieved from a recent publication from our group 86 [28]. Divergence times (in million years ago, mya) were calculated using DT $=\mathrm{Ks} /(2 \times 6.1$

$87 \times 1 e-9) \times 1 e-6$ mya [29].

\subsection{Identification of conserved motifs and cis-regulatory elements}

90 Conserved motifs were identified de novo with the MEME [30] algorithm implemented in the

91 R package universalmotif [31] ( $\operatorname{mmotifs}=10, \operatorname{minw}=6, \operatorname{maxw}=50$ ). Position weight matrices

92 of known cis-regulatory elements (CREs) were downloaded from PlantPAN 3.0 [32]. Promoters

93 were defined as the regions between $-1000 \mathrm{bp}$ and +200 bp relative to the transcription start

94 site, as established for $A$. thaliana [33]. Promoter sequences were scanned for the presence

95 of CREs using the function scan_sequences() from the R package universalmotif [31] with a

96 log-odds threshold of 0.9, and TF families associated with each CRE were retrieved from

97 PlantTFDB [34].

98

\subsection{Gene expression analysis}

100 Raw read counts were downloaded from the Soybean Expression Atlas 101 (http://venanciogroup.uenf.br/cgi-bin/gmax_atlas/index.cgi) [35]. Samples from callus, 102 seedling, endosperm, inflorescences and suspensor were not included in the global expression 103 analysis because they were represented by too few samples $(n<10)$. Additional 98 publicly 104 available RNA-seq BioProjects investigating soybean response to biotic or abiotic stresses 105 (930 samples) were downloaded from NCBI's Sequence Read Archive using our pipeline 106 described in [35] (Supplementary Table S1). A total of 145 pairwise comparisons for 107 differential gene expression were performed with DESeq2 [36] (Supplementary Table S2).

108 Genes were considered differentially expressed if the absolute fold change was greater than 1091.5 and Benjamini-Hochberg-adjusted $P$-value lower than 0.05 . The pairwise comparisons 110 only included samples from the same BioProject to avoid potential artifacts that could bias 111 biological interpretation, such as different time points, cultivars or plant age. 


\section{Results and discussion}

1143.1 Identification, distribution and physicochemical properties of GMPR-1 genes and their 115 protein products

116 Using $A$. thaliana PR-1 proteins as queries, we identified 24 soybean genes encoding PR-1 117 proteins through BLASTp searches, all with a conserved CAP domain (PF00188). Genes were 118 named using the standard nomenclature for other species (GmPR-1-1 to GmPR-1-24; Table 119 1). Most of the GmPR-1 genes were located on chromosomes 13 and 15 ( $n=9$ and 6, 120 respectively), while others were located on chromosomes $7,17,1,2,10$, and $16(n=3,2$, $1211,1,1,1$, respectively). PR-1 genes have been found in widely variable numbers across 122 species, such as tomato [37], grape [38], wheat [39] and rice [40], suggesting that a number 123 of lineage-specific expansions and contractions shaped the PR-1 repertoires across species.

The stress-related functions of GmPR-1 genes are also supported by their associated Gene Ontology terms, which are all related to defense against biotic and abiotic stresses (Supplementary Table S3). A structural analysis revealed that GmPR-1 genes have 8-32 kDa, 79-277 amino acid residues, and their isoelectric points range from 4.3 to 9.1 , with 9 GmPR1 proteins being alkaline and 15 acidic. All GmPR-1 proteins are hydrophilic (GRAVY index $<0)$ and predicted to be secreted to the apoplast, although the signal peptide was not detectable in four of them (Table 1). The median percentage of alpha helices, beta sheets, disordered regions and transmembrane helices were 38,17, 21, and 9, respectively. Overall, our findings are in line with those previously reported for other species $[8,41]$.

3.2 A deletion on the CAP domain removed the caveolin-binding motif and the CAPE peptide in a GmPR-1 gene

136 The multiple sequence alignment of GmPR-1 proteins reveal a high conservation of the CAP domain across sequences (Fig. 1a). The tertiary structures of the GmPR-1 proteins are also conserved, in particular regarding the $\alpha-\beta-\alpha$ sandwich that is distinctive of PR-1 proteins (Fig. 1b) [8]. However, GmPR-1-14 lost a large region of the CAP domain, including the caveolinbinding motif ( $C B M$, responsible for the sterol-binding activity) and the C-terminal region that can be cleaved to release the CAP-derived peptide (CAPE). Expectedly, this deletion

142 significantly affected the tertiary structure of the GmPR-1-14 protein, which differs from the 143 canonical $\alpha-\beta-\alpha$ sandwich and folds into $2 \alpha$-helices linked by a loop (Fig. 1b). 
observation that PR-1 proteins are especially effective against sterol auxotrophs, which are unable to produce their own sterols and depend on sterols from the environment [10]. Additionally, the last 11 amino acid residues of the CAP domain can be cleaved and release a CAP-derived peptide (CAPE), which can act indirectly in plant defense by inducing the expression of several defense-related genes [43]. As GmPR-1-14 lacks both the CBM and the CAPE peptide, this gene has likely lost its antimicrobial properties or it has undergone functional divergence and act in plant defense by some undescribed mechanism.

\subsection{GmPR-1 genes cluster in two phylogenetic groups}

155 We reconstructed the phylogeny of PR-1 genes in soybean, Vigna radiata and $A$. thaliana 156 ( $n=24,8$ and 22, respectively) to understand the evolutionary history of GmPR-1 genes (Fig. 157 2). PR-1 genes from the three species clustered into 3 groups. Cluster 1 comprised the 158 majority of GmPR-1 genes ( $n=13)$, while cluster 2 and 3 contained 2 and 8 GmPR-1 genes, respectively. Cluster 1 comprises mainly genes that arose from recent local duplications, as revealed by their short branch lengths and close genomic location. A similar pattern was observed for AtPR-1 genes, with 4 genes derived from local duplications in cluster 1 . Additionally, most of the GmPR-1 genes in clusters 2 and 3 arose from speciation events in the legume clade, as opposed to cluster 1 , which is dominated by expansions in the soybean lineage after the split from $V$. radiata.

The maximum likelihood phylogenetic tree of GmPR-1 genes was reconstructed using a C. cajan protein (UniProt ID: A0A151S132) as outgroup. The GmPR-1 genes clustered in two groups (Fig. 3a), as observed for grapevine [38] and tomato [37], but not for wheat [39] and rice [40], which were divided in three clusters. The only three GmPR-1 genes that contain introns belong to cluster 1 (Fig. 3b), although these introns were likely acquired independently, as they are in different terminal branches of the phylogenetic tree. The same trend was observed for the position and presence/absence of motifs, from which some are

172 exclusive to cluster 1 , and others co-located near the $\mathrm{N}$-terminus in cluster 2 (Fig. 3c). We

173 also observed that GmPR-1 genes in cluster 2 mostly arose from recent local duplication 174 events, as demonstrated by their shorter branch lengths in comparison to cluster 1. 
177 All GmPR-1 duplicated genes were classified into 5 modes of duplication according to the 178 algorithm described in [44]: dispersed, proximal, tandem, transposed and whole-genome 179 duplications (DD, PD, TD, TRD and WGD, respectively). We found 48 duplicate pairs containing 180 GmPR-1 genes, of which 48\%, 21\%, 13\%, 10\% and 8\% derived from DD, WGD, TD, TRD, 181 and PD, respectively (Supplementary Table S4). A complementary analysis on the PLAZA 182 server revealed that $95 \%$ of the GmPR-1 genes were block duplicates, while $83 \%$ were 183 tandem duplicates.

Local duplications (here referred to as tandem and proximal duplications) represent $21 \%$ of the GmPR-1 duplicate pairs. However, as genes can be duplicated more than once by different modes, this relative frequency is likely underestimated if each gene is assigned to a single pair and duplication mode. Local duplications explain the physical clustering of 9 GmPR1 genes on chromosome 13, of GmPR-1-7 and GmPR-1-8 within a $6.8 \mathrm{~kb}$ region, and of GmPR-1-9 to GmPR-1-15 within a $41.6 \mathrm{~kb}$ region. Likewise, GmPR-1-16 to GmPR-1-21 are clustered in a $26.3 \mathrm{~kb}$ region on chromosome 15 . As a similar trend was observed for AtPR-1 genes (Fig. 2), these findings suggest that independent local duplications have shaped the PR-1 gene repertoire in plants. This physical clustering of defense- and stress-related genes has been described in other species, and it is thought to be an important evolutionary mechanism to ensure coordinated transcriptional regulation $[38,45]$.

The soybean genome has undergone two WGD events, nearly 58 and 13 million years ago, resulting in $75 \%$ of its genes being present in multiple copies [46]. As mentioned above, $21 \%$ of the GmPR-1 genes were generated by WGD events. Most of these genes diverged near the first WGD ( 58 mya) or even in more remote WGDs (Supplementary Table S4). We hypothesize that the $\sim 58$ mya WGD was important to provide the material for the more recent local duplications that resulted in the current soybean GmPR-1 gene repertoire. In addition, our results suggest that duplicates generated upon the 13 mya WGD were subsequently lost, as there are more WGD pairs from older WGDs than from the $~ 13$ mya WGD. We also found that $\mathrm{Ka} / \mathrm{Ks}$ values were lower than 1 for all pairs derived from all modes of duplication, with median values ranging from 0.13 (TD) to 0.24 (TRD) (Fig. 3d), implying that they are under negative selection. Tandem-derived pairs have the lowest $\mathrm{Ka} / \mathrm{Ks}$ values and the narrowest $\mathrm{Ka} / \mathrm{Ks}$ distribution, which indicate that such events happened more recently and around the same time (Fig. 3d). 
We identified 13 TF families encoding potential regulators of GmPR-1 genes. The TF families WRKY, NAC, GATA, HD-Zip, ERF and $\mathrm{C} 2 \mathrm{H} 2$ had detectable binding domains in the regulatory

212 regions of $11,4,3,3,2$ and 1 GmPR-1 genes, respectively (Fig. 3e). C2H2, HD-Zip and NAC

213 TFs are widely known as key regulators of multiple abiotic stresses in plants, including 214 drought, salinity, cold, and heat stress [47-52]. Likewise, ERFs are ethylene-responsive TFs 215 that have been described as hubs in abiotic stress-related gene regulatory networks [53-55]. 216 GATA TFs have been well described in animals and fungi, but their functions in plants remain 217 unclear. Although plant GATA TFs have been linked to photosynthesis-related pathways $218[56,57]$, some studies reported abiotic stress-responsive GATA genes [58,59]. Finally, WRKY 219 TFs are involved in signaling networks activated in abiotic and biotic stresses, particularly in 220 pathogen-associated molecular pattern (PAMP)-triggered immunity $[8,60,61]$. As the most 221 abundant TF binding sites in GmPR-1 promoters are associated with stress-related TF families, 222 our findings suggest that biotic and abiotic stresses are the major stimuli modulating the 223 transcription of GmPR-1 genes.

\subsection{Global expression profiles reveal stress-specific and broadly expressed GmPR-1 genes}

The global expression profiles of GmPR-1 genes in 1972 RNA-seq samples, comprising multiple tissues and conditions, revealed that 23 of 24 GmPR-1 genes are induced by stress conditions, and GmPR-1-13 is exclusively expressed under stress (Fig. 4a). Read counts for GmPR-1-5 are zero or close to zero in almost all samples and greater than 1 in only 17 samples, of which $41 \%$ are related to stress conditions. The distribution of Tau indices for tissue specificity indicates that genes from both phylogenetic groups tend to have tissuespecific expression profiles (i.e., Tau values close to 1) (Supplementary Fig. S1).

PR proteins were originally defined as proteins that are induced only in pathological or related situations [41]. According to van Loon [41], proteins that are homologous to PR proteins but occurred in healthy conditions should be designated as PR-like proteins. Although this classification has been widely overlooked by the scientific community over the years, distinguishing between stress-specific and broadly occurring PR proteins could be useful to identify PR proteins that are exclusively involved in stress response and the ones that can also have endogenous functions. In this sense, GmPR-1-23 is likely the only gene encoding an exclusively stress-related protein, while the majority of GmPR-1 genes might play a role not only in stress response, but also in plant growth and development. Nevertheless, we 
243 immediate first line of response to stress, which would be followed by a stronger, second layer

244 of PR-1 response, harnessed by up-regulation of specific genes.

245 We found that $86 \%(21 / 24)$ of the GmPR-1 genes are up-regulated under at least one 246 abiotic stress condition, such as alkalinity, salt, cadmium, drought, heat, herbicide, high ozone 247 levels, and iron and phosphorus deficiency (Fig. 4b). Similarly, 20/24 GmPR-1 genes are up248 regulated under at least one biotic stress caused by fungi (Fusarium oxysporum, Fusarium 249 virguliforme, Phialophora gregata, and Phakopsora pachyrhizi), insect (Aphis glycines), 250 nematodes (Heterodera glycines, Rotylenchulus reniformis), oomycete (Phytophthora sojae) 251 and virus (soybean mosaic virus) (Fig. 4c). Interestingly, GmPR-1-5 and GmPR-1-14 (CBM252 and CAPE-less protein) were not up-regulated under any of the abiotic and biotic stresses.

Further, the oomycete Phytophthora sojae and the fungus Fusarium virguliforme systematically repressed the expression of GmPR-1 genes, suggesting that these pathogens can down-regulate GmPR-1 transcription. Conversely, GmPR-1-1, GmPR-1-16 and GmPR-118 were up-regulated in the greatest number of biotic stress conditions $(n=6,6,5$, respectively), while $G m P R-1-7, G m P R-1-21, G m P R-1-22$ were up-regulated in the greatest number of abiotic stress conditions ( $n=5$ for all). Thus, these six genes are the most promising genes for biotechnological applications aimed at increasing soybean resistance to biotic and abiotic stresses, respectively.

Little is known about the transcriptional landscape of PR-1 genes in response to various stress conditions, in particular because the studies that investigated the expression profiles of such genes are often limited to a few stress conditions. For instance, PR-1 genes were upregulated under drought stress in tomato [37] and upon challenge with the fungal pathogen Septoria nodorum in wheat [39]. Our findings represent the most comprehensive analysis of the transcriptional responses of PR-1 genes to biotic and abiotic stresses, with 26 different stress conditions. Our results highlight stress-responsive GmPR-1 genes that are potential targets for plant transformation and development of resistant cultivars.

\section{Conclusions}

271 Here, we reported the soybean PR-1 gene repertoire and characterized it at the sequence,

272 structural and transcriptional levels. The 24 GmPR-1 genes clustered in two phylogenetic 273 groups and are constrained by strong purifying selection. Overall, GmPR-1 proteins are highly 274 conserved in primary and tertiary structures. Further, upstream of GmPR-1 genes, we found 275 multiple binding sites of major stress-related TF families, namely WRKY, HD-ZIP, NAC, ERF, 
$276 \mathrm{C} 2 \mathrm{H} 2$ and GATA. Finally, we found that the vast majority of GmPR-1 genes are up-regulated 277 under stress conditions, helping uncover candidate genes to be used in the development of 278 soybean lines resistant to both biotic and abiotic stresses.

280 Data statement

281 All data and code used in this study are available in our Figshare repository (DOI: $28210.6084 / \mathrm{m9}$.figshare.14330354) to ensure full reproducibility.

\section{Acknowledgements}

285 This work was supported by Fundação Carlos Chagas Filho de Amparo à Pesquisa do Estado 286 do Rio de Janeiro (FAPERJ; grants E-26/203.309/2016 and E-26/203.014/2018), 287 Coordenação de Aperfeiçoamento de Pessoal de Nível Superior - Brasil (CAPES; Finance Code 288 001), and Conselho Nacional de Desenvolvimento Científico e Tecnológico. The funding 289 agencies had no role in the design of the study and collection, analysis, and interpretation of 290 data and in writing.

\section{Author contributions}

293 Conceived the study: FA-S and TMV. Data analysis: FA-S. Funding, project coordination and 294 infrastructure: TMV. Manuscript writing: FA-S and TMV. 


\section{REFERENCES}

[1] L.C. Van Loon, M. Rep, C.M.J. Pieterse, Significance of inducible defense-related proteins in infected plants, Annu. Rev. Phytopathol. 44 (2006) 135-162. https://doi.org/10.1146/annurev.phyto.44.070505.143425.

[2] D. Alexander, R.M. Goodman, M. Gut-Rella, C. Glascock, K. Weymann, L. Friedrich, D. Maddox, P. Ahl-Goy, T. Luntz, E. Ward, Increased tolerance to two oomycete pathogens in transgenic tobacco expressing pathogenesis-related protein 1a, Proc. Natl. Acad. Sci. 90 (1993) 7327 LP - 7331. https://doi.org/10.1073/pnas.90.15.7327.

[3] C.B. Lawrence, M.H.A.J. Joosten, S. Tuzun, Differential induction of pathogenesisrelated proteins in tomato by $<i>$ Alternaria solani and the association of a basic chitinase isozyme with resistance, Physiol. Mol. Plant Pathol. 48 (1996) 361-377. https://doi.org/https://doi.org/10.1006/pmpp.1996.0029.

[4] K.S. Kothari, P.K. Dansana, J. Giri, A.K. Tyagi, Rice Stress Associated Protein 1 (OsSAP1) Interacts with Aminotransferase (OsAMTR1) and Pathogenesis-Related 1a Protein (OsSCP) and Regulates Abiotic Stress Responses , Front. Plant Sci. . 7 (2016) 1057.

[5] S.H. Shin, J.-H. Pak, M.J. Kim, H.J. Kim, J.S. Oh, H.K. Choi, H.W. Jung, Y.S. Chung, An Acidic PATHOGENESIS-RELATED1 Gene of Oryza grandiglumis is Involved in Disease Resistance Response Against Bacterial Infection, Plant Pathol. J. 30 (2014) 208-214. https://doi.org/10.5423/PPJ.NT.11.2013.0112.

[6] R.S.S. Fraser, Evidence for the occurrence of the "pathogenesis-related" proteins in leaves of healthy tobacco plants during flowering, Physiol. Plant Pathol. 19 (1981) 6976. https://doi.org/https://doi.org/10.1016/S0048-4059(81)80009-4.

[7] G. Sessa, X.-Q. Yang, V. Raz, Y. Eyal, R. Fluhr, Dark induction and subcellular localization of the pathogenesis-related PRB-1b protein, Plant Mol. Biol. 28 (1995) 537547. https://doi.org/10.1007/BF00020400.

[8] S. Breen, S.J. Williams, M. Outram, B. Kobe, P.S. Solomon, Emerging Insights into the Functions of Pathogenesis-Related Protein 1, Trends Plant Sci. 22 (2017) 871-879. https://doi.org/10.1016/j.tplants.2017.06.013.

[9] P.J. Seo, A.-K. Lee, F. Xiang, C.-M. Park, Molecular and Functional Profiling of Arabidopsis Pathogenesis-Related Genes: Insights into Their Roles in Salt Response of 
Seed Germination, Plant Cell Physiol. $49 \quad$ (2008) 334-344. https://doi.org/10.1093/pcp/pcn011.

[10] T. Lotan, N. Ori, R. Fluhr, Pathogenesis-related proteins are developmentally regulated in tobacco flowers., Plant Cell. $1 \quad$ (1989) $881 \quad$ LP $\quad$ - 887. https://doi.org/10.1105/tpc.1.9.881.

[11] A.Y. Bandara, D.K. Weerasooriya, C.A. Bradley, T.W. Allen, P.D. Esker, Dissecting the economic impact of soybean diseases in the United States over two decades, PLoS One. 15 (2020) 1-28. https://doi.org/10.1371/journal.pone.0231141.

[12] F.A. Rodrigues, R. Fuganti-Pagliarini, J. Marcolino-Gomes, T.J. Nakayama, H.B.C. Molinari, F.P. Lobo, F.G. Harmon, A.L. Nepomuceno, Daytime soybean transcriptome fluctuations during water deficit stress, BMC Genomics. 16 (2015) 505. https://doi.org/10.1186/s12864-015-1731-x.

[13] A. Kiba, M. Nishihara, T. Nakatsuka, S. Yamamura, Pathogenesis-related protein 1 homologue is an antifungal protein in Wasabia japonica leaves and confers resistance to Botrytis cinerea in transgenic tobacco, Plant Biotechnol. 24 (2007) 247-253. https://doi.org/10.5511/plantbiotechnology.24.247.

[14] S. Sarowar, Y.J. Kim, E.N. Kim, K.D. Kim, B.K. Hwang, R. Islam, J.S. Shin, Overexpression of a pepper basic pathogenesis-related protein $1 \mathrm{gene}$ in tobacco plants enhances resistance to heavy metal and pathogen stresses, Plant Cell Rep. 24 (2005) 216-224. https://doi.org/10.1007/s00299-005-0928-x.

[15] M. Van Bel, T. Diels, E. Vancaester, L. Kreft, A. Botzki, Y. Van De Peer, F. Coppens, K. Vandepoele, PLAZA 4.0: An integrative resource for functional, evolutionary and comparative plant genomics, Nucleic Acids Res. 46 (2018) D1190-D1196. https://doi.org/10.1093/nar/gkx1002.

[16] S.F. Altschul, W. Gish, W. Miller, E.W. Myers, D.J. Lipman, Basic local alignment search tool, J. Mol. Biol. 215 (1990) 403-410. https://doi.org/https://doi.org/10.1016/S00222836(05)80360-2.

[17] A. V Brown, S.I. Conners, W. Huang, A.P. Wilkey, D. Grant, N.T. Weeks, S.B. Cannon, M.A. Graham, R.T. Nelson, A new decade and new data at SoyBase, the USDA-ARS soybean genetics and genomics database, Nucleic Acids Res. 13 (2020) 1-6. https://doi.org/10.1093/nar/gkaa1107. 
[18] L.A. Kelley, S. Mezulis, C.M. Yates, M.N. Wass, M.J. Sternberg, The Phyre2 web portal for protein modeling, prediction and analysis, Nat. Protoc. 10 (2016) 845-858. https://doi.org/10.1038/nprot.2015-053.

[19] D. Osorio, P. Rondón-Villarrea, R. Torres, Peptides: a package for data mining of antimicrobial peptides., R J. 7 (2015).

[20] J.J.A. Armenteros, K.D. Tsirigos, C.K. Sønderby, T.N. Petersen, O. Winther, S. Brunak, G. von Heijne, H. Nielsen, SignalP 5.0 improves signal peptide predictions using deep neural networks, Nat. Biotechnol. 37 (2019) 420-423.

[21] D. Wilkins, Z. Kurtz, gggenes: Draw gene arrow maps in "ggplot2," R Packag. Version 0.4. 0. (2019).

[22] H. Pages, P. Aboyoun, R. Gentleman, S. DebRoy, Biostrings: String objects representing biological sequences, and matching algorithms, R Packag. Version. 2 (2016) 10-18129.

[23] K. Katoh, K. Misawa, K. Kuma, T. Miyata, MAFFT: a novel method for rapid multiple sequence alignment based on fast Fourier transform, Nucleic Acids Res. 30 (2002) 3059-3066. https://doi.org/10.1093/nar/gkf436.

[24] U. Bodenhofer, E. Bonatesta, C. Horejš-Kainrath, S. Hochreiter, msa: an R package for multiple sequence alignment, Bioinformatics. $31 \quad$ (2015) 3997-3999. https://doi.org/10.1093/bioinformatics/btv494.

[25] B.Q. Minh, H.A. Schmidt, O. Chernomor, D. Schrempf, M.D. Woodhams, A. von Haeseler, R. Lanfear, IQ-TREE 2: New Models and Efficient Methods for Phylogenetic Inference in the Genomic Era, Mol. Biol. Evol. 37 (2020) 1530-1534. https://doi.org/10.1093/molbev/msaa015.

[26] T.U. Consortium, UniProt: a worldwide hub of protein knowledge, Nucleic Acids Res. 47 (2019) D506-D515. https://doi.org/10.1093/nar/gky1049.

[27] G. Yu, D.K. Smith, H. Zhu, Y. Guan, T.T.-Y. Lam, ggtree: an r package for visualization and annotation of phylogenetic trees with their covariates and other associated data, Methods Ecol. Evol. 8 (2017) 28-36. https://doi.org/https://doi.org/10.1111/2041210X.12628.

[28] F. Almeida-Silva, K. Moharana, F. Machado, T. Venancio, Exploring the complexity of soybean ( Glycine max ) transcriptional regulation using global gene co-expression 
networks, Planta. (2020) 1-12. https://doi.org/10.1101/2020.06.19.161950.

[29] J. Guo, C. Li, X. Zhang, Y. Li, D. Zhang, Y. Shi, Y. Song, Y. Li, D. Yang, T. Wang, Transcriptome and GWAS analyses reveal candidate gene for seminal root length of maize seedlings under drought stress, Plant Sci. 292 (2020). https://doi.org/10.1016/j.plantsci.2019.110380.

[30] T.L. Bailey, M. Boden, F.A. Buske, M. Frith, C.E. Grant, L. Clementi, J. Ren, W.W. Li, W.S. Noble, MEME Suite: Tools for motif discovery and searching, Nucleic Acids Res. 37 (2009) 202-208. https://doi.org/10.1093/nar/gkp335.

[31] B.J.-M. Tremblay, universalmotif: Import, Modify, and Export Motifs with R, (2020).

[32] C.N. Chow, T.Y. Lee, Y.C. Hung, G.Z. Li, K.C. Tseng, Y.H. Liu, P.L. Kuo, H.Q. Zheng, W.C. Chang, Plantpan3.0: A new and updated resource for reconstructing transcriptional regulatory networks from chip-seq experiments in plants, Nucleic Acids Res. 47 (2019) D1155-D1163. https://doi.org/10.1093/nar/gky1081.

[33] C.P. Yu, J.J. Lin, W.H. Li, Positional distribution of transcription factor binding sites in Arabidopsis thaliana, Sci. Rep. 6 (2016) 1-7. https://doi.org/10.1038/srep25164.

[34] J. Jin, F. Tian, D.C. Yang, Y.Q. Meng, L. Kong, J. Luo, G. Gao, PlantTFDB 4.0: toward a central hub for transcription factors and regulatory interactions in plants, Nucleic Acids Res. 45 (2017) D1040-D1045. https://doi.org/10.1093/nar/gkw982.

[35] F.B. Machado, K.C. Moharana, F. Almeida-Silva, R.K. Gazara, F. Pedrosa-Silva, F.S. Coelho, C. Grativol, T.M. Venancio, Systematic analysis of 1,298 RNA-Seq samples and construction of a comprehensive soybean ( Glycine max ) expression atlas, Plant J. (2020) 0-2. https://doi.org/10.1111/tpj.14850.

[36] M.I. Love, W. Huber, S. Anders, Moderated estimation of fold change and dispersion for RNA-seq data with DESeq2, Genome Biol. 15 (2014) 1-21. https://doi.org/10.1186/s13059-014-0550-8.

[37] M.A. Akbudak, S. Yildiz, E. Filiz, Pathogenesis related protein-1 (PR-1) genes in tomato (Solanum lycopersicum L.): Bioinformatics analyses and expression profiles in response to drought stress, Genomics. $112 \quad$ (2020) 4089-4099. https://doi.org/10.1016/j.ygeno.2020.07.004.

[38] Z.T. Li, S.A. Dhekney, D.J. Gray, PR-1 gene family of grapevine: A uniquely duplicated PR-1 gene from a Vitis interspecific hybrid confers high level resistance to bacterial 
disease in transgenic tobacco, Plant Cell Rep. 30 (2011) 1-11. https://doi.org/10.1007/s00299-010-0934-5.

[39] S. Lu, T.L. Friesen, J.D. Faris, Molecular characterization and genomic mapping of the pathogenesis-related protein 1 (PR-1) gene family in hexaploid wheat (Triticum aestivum L.), Mol. Genet. Genomics. 285 (2011) 485-503. https://doi.org/10.1007/s00438-011-0618-z.

[40] Q. Liu, Q. Xue, Computational identification of novel PR-1-type genes in Oryza sativa, J. Genet. 85 (2006) 193-198. https://doi.org/10.1007/BF02935330.

[41] L.C. van Loon, W.S. Pierpoint, T. Boller, V. Conejero, Recommendations for naming plant pathogenesis-related proteins, Plant Mol. Biol. Report. 12 (1994) 245-264. https://doi.org/10.1007/BF02668748.

[42] J. Gamir, R. Darwiche, P. van't Hof, V. Choudhary, M. Stumpe, R. Schneiter, F. Mauch, The sterol-binding activity of PATHOGENESIS-RELATED PROTEIN 1 reveals the mode of action of an antimicrobial protein, Plant J. 89 (2017) 502-509. https://doi.org/10.1111/tpj.13398.

[43] Y.-L. Chen, C.-Y. Lee, K.-T. Cheng, W.-H. Chang, R.-N. Huang, H.G. Nam, Y.-R. Chen, Quantitative Peptidomics Study Reveals That a Wound-Induced Peptide from PR-1 Regulates Immune Signaling in Tomato, Plant Cell. 26 (2014) 4135 LP - 4148. https://doi.org/10.1105/tpc.114.131185.

[44] X. Qiao, Q. Li, H. Yin, K. Qi, L. Li, R. Wang, S. Zhang, A.H. Paterson, Gene duplication and evolution in recurring polyploidization - diploidization cycles in plants, Genome Biol. (2019) 1-23.

[45] G. Amoutzias, Y. Van de Peer, Together We Stand: Genes Cluster to Coordinate Regulation, Dev. Cell. $14 \quad$ 640-642. https://doi.org/10.1016/j.devcel.2008.04.006.

[46] J. Schmutz, S.B. Cannon, J. Schlueter, J. Ma, T. Mitros, W. Nelson, D.L. Hyten, Q. Song, J.J. Thelen, J. Cheng, D. Xu, U. Hellsten, G.D. May, B. Valliyodan, E. Lindquist, M. Peto, D. Grant, S. Shu, D. Goodstein, K. Barry, M. Futrell-griggs, B. Abernathy, J. Du, Z. Tian, L. Zhu, N. Gill, T. Joshi, M. Libault, A. Sethuraman, X. Zhang, K. Shinozaki, H.T. Nguyen, R.A. Wing, P. Cregan, J. Specht, J. Grimwood, D. Rokhsar, G. Stacey, R.C. Shoemaker, S.A. Jackson, Genome sequence of the palaeopolyploid soybean, Nature. 463 (2010) 178-183. https://doi.org/10.1038/nature08670. 
[47] M. Van Bel, K. Vandepoele, Comment on 'Hayai-Annotation Plants: an ultrafast and comprehensive functional gene annotation system in plants': the importance of taking the GO graph structure into account, Bioinformatics. (2020) 1-3. https://doi.org/10.1093/bioinformatics/btaa1052.

[48] N. Zhao, X. Ding, T. Lian, M. Wang, Y. Tong, D. Liang, Q. An, S. Sun, S.A. Jackson, B. Liu, C. Xu, The Effects of Gene Duplication Modes on the Evolution of Regulatory Divergence in Wild and Cultivated Soybean, Front. Genet. 11 (2020) 1-9. https://doi.org/10.3389/fgene.2020.601003.

[49] A.A. Golicz, P.E. Bayer, P.L. Bhalla, J. Batley, D. Edwards, Pangenomics Comes of Age: From Bacteria to Plant and Animal Applications, Trends Genet. 36 (2020) 132-145. https://doi.org/10.1016/j.tig.2019.11.006.

[50] X. Dai, Z. Zhuang, C. Boschiero, Y. Dong, P.X. Zhao, LegumeIP V3: from models to crops-an integrative gene discovery platform for translational genomics in legumes, Nucleic Acids Res. 49 (2021) D1472-D1479. https://doi.org/10.1093/nar/gkaa976.

[51] S. Gong, Y. Ding, S. Hu, L. Ding, Z. Chen, C. Zhu, The role of HD-Zip class I transcription factors in plant response to abiotic stresses, Physiol. Plant. 167 (2019) 516-525. https://doi.org/10.1111/ppl.12965.

[52] W. Shen, H. Li, R. Teng, Y. Wang, W. Wang, J. Zhuang, Genomic and transcriptomic analyses of HD-Zip family transcription factors and their responses to abiotic stress in tea plant (Camellia sinensis), Genomics. $111 \quad$ (2019) 1142-1151. https://doi.org/10.1016/j.ygeno.2018.07.009.

[53] J. Mizoi, K. Shinozaki, K. Yamaguchi-Shinozaki, AP2/ERF family transcription factors in plant abiotic stress responses, Biochim. Biophys. Acta - Gene Regul. Mech. 1819 (2012) 86-96. https://doi.org/10.1016/j.bbagrm.2011.08.004.

[54] Z. Xie, T.M. Nolan, H. Jiang, Y. Yin, AP2/ERF transcription factor regulatory networks in hormone and abiotic stress responses in Arabidopsis, Front. Plant Sci. 10 (2019) 117. https://doi.org/10.3389/fpls.2019.00228.

[55] M. Müller, S. Munné-Bosch, Ethylene response factors: A key regulatory hub in hormone and stress signaling, Plant Physiol. 169 (2015) 32-41. https://doi.org/10.1104/pp.15.00677.

[56] C. Zhang, Y. Huang, Z. Xiao, H. Yang, Q. Hao, S. Yuan, H. Chen, L. Chen, S. Chen, X. 
Zhou, W. Huang, A GATA Transcription Factor from Soybean (Glycine max) Regulates Chlorophyll Biosynthesis and Suppresses Growth in the Transgenic Arabidopsis thaliana, Plants . 9 (2020). https://doi.org/10.3390/plants9081036.

[57] Y. An, Y. Zhou, X. Han, C. Shen, S. Wang, C. Liu, W. Yin, X. Xia, The GATA transcription factor GNC plays an important role in photosynthesis and growth in poplar, J. Exp. Bot. 71 (2020) 1969-1984. https://doi.org/10.1093/jxb/erz564.

[58] K.K. Nutan, S.L. Singla-Pareek, A. Pareek, The Saltol QTL-localized transcription factor OsGATA8 plays an important role in stress tolerance and seed development in Arabidopsis and rice, J. Exp. Bot. 71 (2020) 684-698. https://doi.org/10.1093/jxb/erz368.

[59] P. Gupta, K.K. Nutan, S.L. Singla-Pareek, A. Pareek, Abiotic Stresses Cause Differential Regulation of Alternative Splice Forms of GATA Transcription Factor in Rice , Front. Plant Sci. . 8 (2017) 1944.

[60] C. Villano, S. Esposito, V. D’Amelia, R. Garramone, D. Alioto, A. Zoina, R. Aversano, D. Carputo, WRKY genes family study reveals tissue-specific and stress-responsive TFs in wild potato species, Sci. Rep. 10 (2020) 1-12. https://doi.org/10.1038/s41598-02063823-w.

[61] J. Jiang, S. Ma, N. Ye, M. Jiang, J. Cao, J. Zhang, WRKY transcription factors in plant responses to stresses, J. Integr. Plant Biol. 59 (2017) 86-101. https://doi.org/10.1111/jipb.12513. 
bioRxiv preprint doi: https://doi.org/10.1101/2021.03.27.437342; this version posted March 29, 2021. The copyright holder for this preprint (which was not certified by peer review) is the author/funder, who has granted bioRxiv a license to display the preprint in perpetuity. It is made available under aCC-BY-NC-ND 4.0 International license.

\section{Figures}

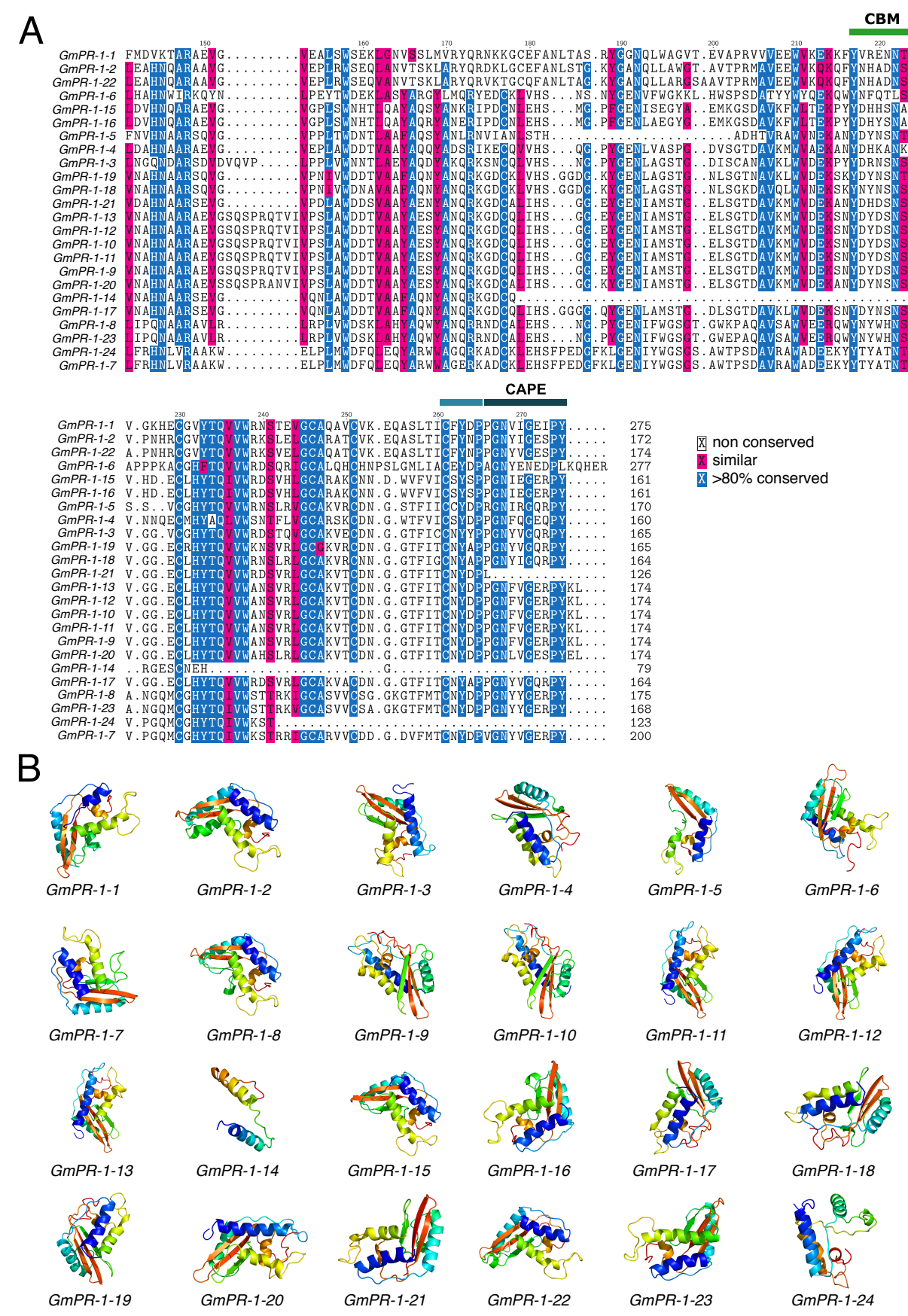

Fig. 1. Multiple sequence alignment and predicted tertiary structures of GmPR-1 proteins. (A) Multiple sequence alignment of the CAP domain. CBM, caveolin-binding motif (responsible for sterol binding). CAPE, CAP-derived peptide. Light blue, CAPE cleavage motif. (B) Tertiary structures of GmPR-1 proteins in amino to carboxy rainbow color key. Blue represents $\mathrm{N}$-terminus and red represents $\mathrm{C}$-terminus. For the correspondence between gene IDs (Wm82.a2.v1) and gene names, see Table 1. 


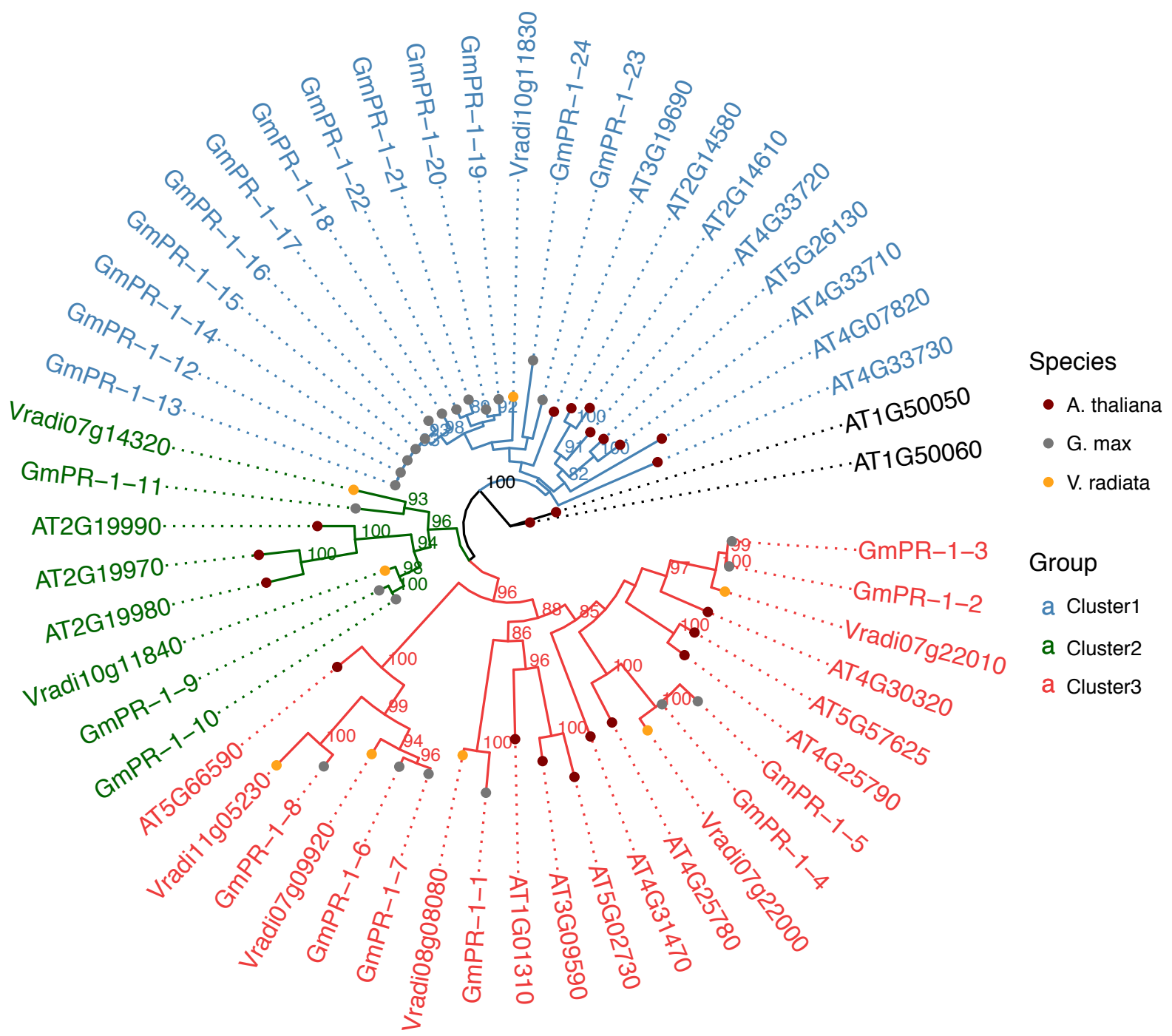

Fig. 2. Phylogenetic reconstruction of PR-1 proteins from Glycine max, Vigna radiata and Arabidopsis thaliana. The unrooted phylogenetic tree was inferred with IQTREE2. Bootstrap support values $>80$ are shown in nodes. Different species and different phylogenetic clusters are represented by distinct tip colors and branch colors, respectively. 

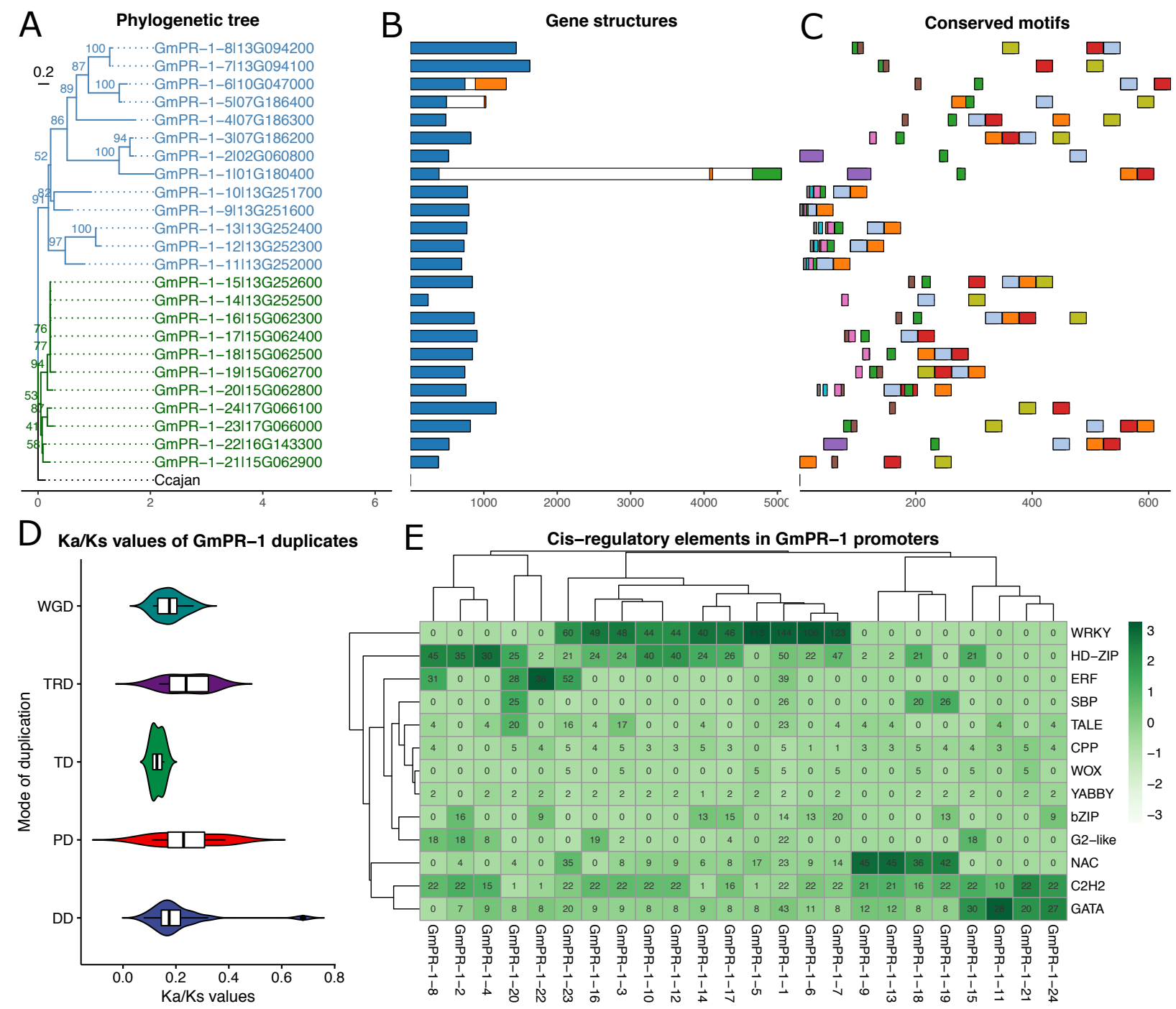

$\mathrm{E}$

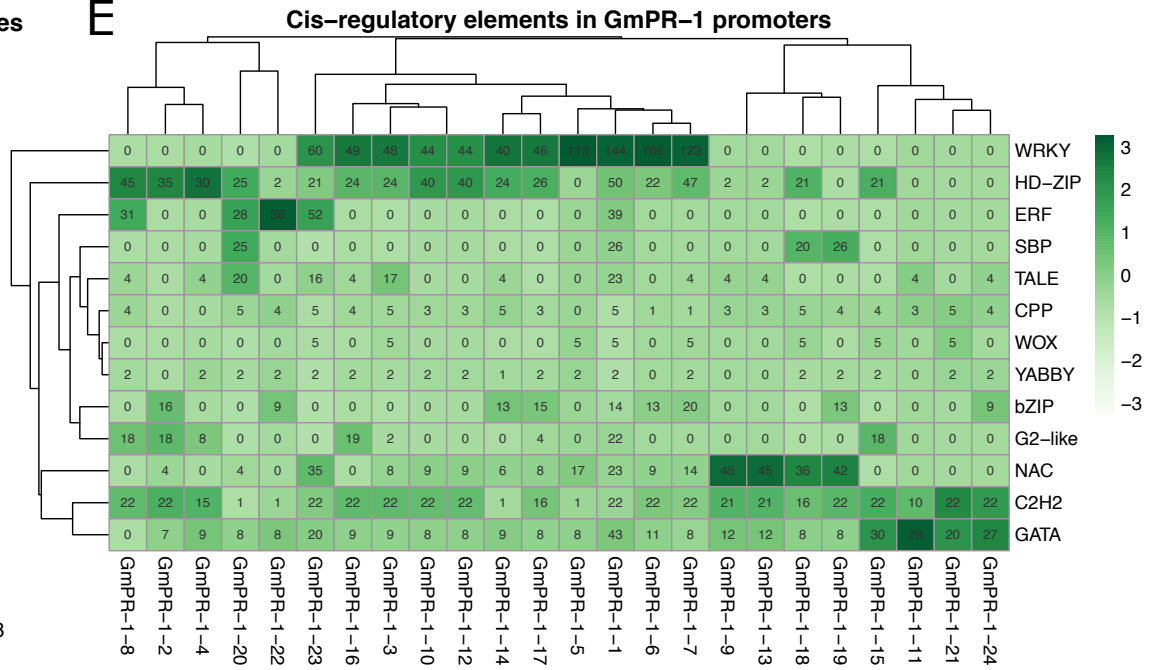

Fig. 3. Genomic properties of GmPR-1 genes. (A) Maximum-likelihood tree inferred with IQTREE2, with 1000 bootstraps and a Cajanus cajan sequence (UniProt ID: A0A151S132) used as outgroup. Bootstrap support values are shown in nodes. Partial Wm82.a2.v1 gene IDs are represented after each gene's name to emphasize the close genomic positions. (B) Gene structures demonstrating exon-intron boundaries. (C) Conserved motifs identified de novo with MEME. (D) Ratio of the number of nonsynonymous substitutions per nonsynonymous substitution site $(\mathrm{Ka})$ to the number of synonymous substitutions per synonymous substitution site (Ks). WGD, whole-genome duplication. TRD, transposed duplication. TD, tandem duplication. PD, proximal duplication. DD, dispersed duplication. (E) Heatmap of absolute frequency of known cis-regulatory elements (CREs) in promoter sequences of GmPR-1 genes. Genes and samples were hierarchically clustered based on the matrix of CRE frequencies. Color intensities are scaled by column to highlight the most abundant CREs for each GmPR-1 gene. 
bioRxiv preprint doi: https://doi.org/10.1101/2021.03.27.437342; this version posted March 29, 2021. The copyright holder for this preprint (which was not certified by peer review) is the author/funder, who has granted bioRxiv a license to display the preprint in perpetuity. It is made available under aCC-BY-NC-ND 4.0 International license.

A Global expression profile of GmPR-1 genes

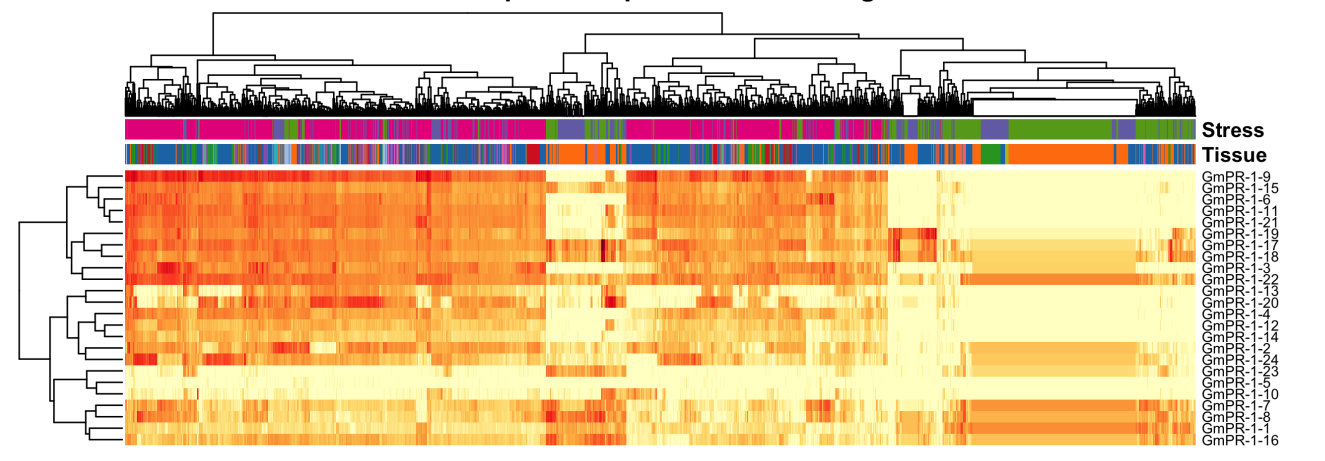

B

GmPR-1 response to abiotic stress

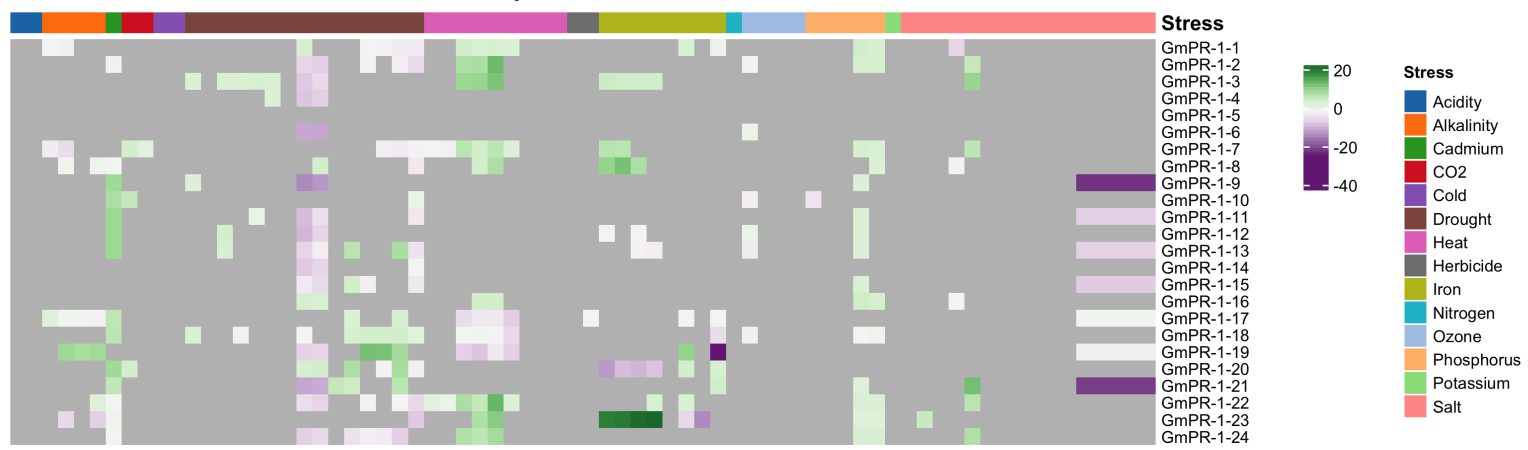

C

GmPR-1 response to biotic stress

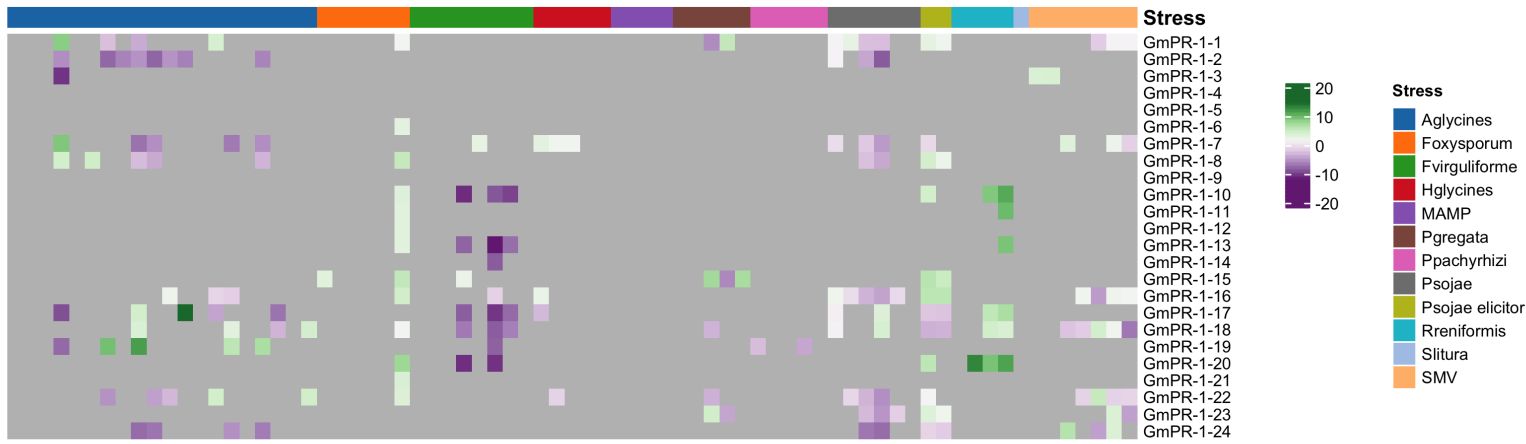

Fig. 4. Global and stress-induced expression profiles of GmPR-1 genes. (A) $\log _{2}$ read counts across different tissues and conditions. Dendrograms represent hierarchical clustering of genes and samples. (B) and (C) $\log _{2}$ fold change of GmPR-1 genes in response to abiotic and biotic stress, respectively. Grey represents unchanged expression, green represents up-regulation and purple represents downregulation. Genes are considered differentially expressed if absolute fold change $>1.5$ and adjusted $P$ value (Benjamini-Hochberg) $<0.05$. 
bioRxiv preprint doi: $\mathrm{https}$ //doi.org/10.1101/2021.03.27.437342; this version posted March 29,2021 . The copyright holder for this preprint (which was not certified by peer review) is the author/funder, who has granted bioRxiv a license to display the preprint in perpetuity. It is made available under aCC-BY-NC-ND 4.0 International license.

\section{Tables}

Table 1. Structural properties of the identified GmPR-1 genes.

\begin{tabular}{|c|c|c|c|c|c|c|c|c|c|c|c|c|}
\hline Gene ID & Gene name & $\begin{array}{l}\text { Exon } \\
\text { no. }\end{array}$ & $\begin{array}{l}\text { Length } \\
\text { (aa) }\end{array}$ & $\mathrm{pI}$ & $\begin{array}{l}\text { MW } \\
(\mathrm{kDa})\end{array}$ & $\mathrm{H}$ & SL & $\mathrm{SP}$ & $\begin{array}{l}\mathrm{AH} \\
\%\end{array}$ & $\begin{array}{l}\text { BS } \\
\%\end{array}$ & $\begin{array}{l}\mathrm{D} \\
\%\end{array}$ & $\begin{array}{l}\mathrm{TH} \\
\%\end{array}$ \\
\hline Glyma.01G180400 & GmPR-1-1 & 3 & 275 & 7.2 & 30 & -0.17 & Apoplast & - & 26 & 21 & 28 & 0 \\
\hline Glyma.02G060800 & GmPR-1-2 & 1 & 172 & 7.4 & 19 & -0.21 & Apoplast & $21-22$ & 35 & 16 & 22 & 0 \\
\hline Glyma.07G186200 & GmPR-1-3 & 1 & 165 & 6 & 18 & -0.38 & Apoplast & $25-26$ & 38 & 19 & 19 & 10 \\
\hline Glyma.07G186300 & GmPR-1-4 & 1 & 160 & 4.9 & 18 & -0.19 & Apoplast & $23-24$ & 39 & 18 & 19 & 0 \\
\hline Glyma.07G186400 & GmPR-1-5 & 2 & 170 & 9.1 & 19 & -0.41 & Apoplast & - & 36 & 13 & 28 & 9 \\
\hline Glyma.10G047000 & GmPR-1-6 & 2 & 277 & 8.5 & 32 & -0.84 & Apoplast & $22-23$ & 22 & 13 & 48 & 0 \\
\hline Glyma.13G094100 & GmPR-1-7 & 1 & 200 & 6.9 & 23 & -0.36 & Apoplast & $22-23$ & 32 & 16 & 32 & 8 \\
\hline Glyma.13G094200 & GmPR-1-8 & 1 & 175 & 9.1 & 20 & -0.37 & Apoplast & $25-26$ & 39 & 15 & 25 & 9 \\
\hline Glyma.13G251600 & GmPR-1-9 & 1 & 174 & 4.3 & 19 & -0.15 & Apoplast & $27-28$ & 38 & 17 & 21 & 9 \\
\hline Glyma.13G251700 & GmPR-1-10 & 1 & 174 & 4.3 & 19 & -0.15 & Apoplast & $27-28$ & 38 & 17 & 21 & 9 \\
\hline Glyma.13G252000 & GmPR-1-11 & 1 & 174 & 4.3 & 19 & -0.15 & Apoplast & $27-28$ & 38 & 17 & 21 & 9 \\
\hline Glyma.13G252300 & GmPR-1-12 & 1 & 174 & 4.3 & 19 & -0.15 & Apoplast & $27-28$ & 38 & 17 & 21 & 9 \\
\hline Glyma.13G252400 & GmPR-1-13 & 1 & 174 & 4.3 & 19 & -0.15 & Apoplast & $27-28$ & 38 & 17 & 21 & 9 \\
\hline Glyma.13G252500 & GmPR-1-14 & 1 & 79 & 5.5 & 8 & -0.05 & Apoplast & $25-26$ & 59 & 3 & 23 & 0 \\
\hline Glyma.13G252600 & GmPR-1-15 & 1 & 161 & 6.1 & 18 & -0.32 & Apoplast & $25-26$ & 40 & 16 & 14 & 10 \\
\hline Glyma.15G062300 & GmPR-1-16 & 1 & 161 & 5.8 & 18 & -0.34 & Apoplast & $25-26$ & 40 & 17 & 17 & 10 \\
\hline Glyma.15G062400 & GmPR-1-17 & 1 & 164 & 5.1 & 18 & -0.18 & Apoplast & $26-27$ & 39 & 21 & 15 & 10 \\
\hline Glyma.15G062500 & GmPR-1-18 & 1 & 164 & 8.4 & 18 & -0.26 & Apoplast & $26-27$ & 38 & 20 & 15 & 10 \\
\hline Glyma.15G062700 & GmPR-1-19 & 1 & 165 & 8 & 18 & -0.33 & Apoplast & $27-28$ & 39 & 18 & 17 & 10 \\
\hline Glyma.15G062800 & GmPR-1-20 & 1 & 174 & 4.4 & 19 & -0.06 & Apoplast & $27-28$ & 40 & 17 & 20 & 9 \\
\hline Glyma.15G062900 & GmPR-1-21 & 1 & 126 & 4.4 & 14 & -0.4 & Apoplast & - & 35 & 25 & 12 & 0 \\
\hline Glyma.16G143300 & GmPR-1-22 & 1 & 174 & 8.8 & 19 & -0.11 & Apoplast & $21-22$ & 36 & 16 & 21 & 9 \\
\hline Glyma.17G066000 & GmPR-1-23 & 1 & 168 & 8.7 & 19 & -0.45 & Apoplast & $17-18$ & 37 & 17 & 22 & 0 \\
\hline Glyma.17G066100 & GmPR-1-24 & 1 & 123 & 6.9 & 15 & -0.61 & Apoplast & - & 43 & 11 & 19 & 0 \\
\hline
\end{tabular}

H: GRAVY hydrophobicity index based on the Kyte-Doolittle scale; SL: Subcellular localization; SP: Signal peptide cleavage site; AH, BS, D and TH: Predicted percentage of alfa-helices, beta-sheets, disordered regions and transmembrane helices in the protein structure. 